Research Article

\title{
A note on the identification numbers of caterpillars
}

\author{
Yuya Kono, Ping Zhang* \\ Department of Mathematics, Western Michigan University, Kalamazoo, Michigan 49008-5248, USA
}

(Received: 21 July 2021. Received in revised form: 20 September 2021. Accepted: 20 September 2021. Published online: 23 September 2021.)

(c) 2021 the authors. This is an open access article under the CC BY (International 4.0) license (www.creativecommons.org/licenses/by/4.0/).

\begin{abstract}
A red-white coloring of a nontrivial connected graph $G$ of diameter $d$ is an assignment of red and white colors to the vertices of $G$ where at least one vertex is colored red. Associated with each vertex $v$ of $G$ is a $d$-vector, called the code of $v$, whose $i$ th coordinate is the number of red vertices at distance $i$ from $v$. A red-white coloring of $G$ for which distinct vertices have distinct codes is called an identification coloring or ID-coloring of $G$. A graph $G$ possessing an ID-coloring is an ID-graph. The minimum number of red vertices among all ID-colorings of an ID-graph $G$ is the identification number or ID-number of $G$. A caterpillar is a tree of order 3 or more, the removal of whose leaves produces a path. A caterpillar possessing an ID-coloring is an ID-caterpillar. In this note, we characterize all ID-caterpillars, determine all possible values of the ID-numbers of ID-caterpillars, and show that each value is realizable.
\end{abstract}

Keywords: distance; ID-coloring; ID-number; ID-graph; caterpillar.

2020 Mathematics Subject Classification: 05C05, 05C12, 05C15, $05 \mathrm{C} 90$.

\section{Introduction}

Let $G$ be a nontrivial connected graph. The distance $d(u, v)$ between vertices $u$ and $v$ in $G$ is the minimum number of edges in a $u-v$ path in $G$. The eccentricity $e(v)$ of a vertex $v$ of $G$ is the distance between $v$ and a vertex farthest from $v$ in $G$. The diameter $\operatorname{diam}(G)$ of $G$ is the largest eccentricity among the vertices of $G$. Equivalently, the diameter of $G$ is the greatest distance between any two vertices of $G$.

Let $G$ be a connected graph of diameter $d \geq 2$ and let there be given a red-white vertex coloring $c$ of $G$ where at least one vertex is colored red. That is, the color $c(v)$ of a vertex $v$ in $G$ is either red or white and $c(v)$ is red for at least one vertex $v$ of $G$. With each vertex $v$ of $G$, there is associated a d-vector $\vec{d}(v)=\left(a_{1}, a_{2}, \ldots, a_{d}\right)$ called the code of $v$ corresponding to $c$, where the $i$ th coordinate $a_{i}$ is the number of red vertices at distance $i$ from $v$ for $1 \leq i \leq d$. If distinct vertices of $G$ have distinct codes, then $c$ is called an identification coloring or ID-coloring of $G$. A graph possessing an identification coloring is an ID-graph. The minimum number of red vertices among all ID-colorings of an ID-graph $G$ is the identification number or ID-number $\operatorname{ID}(G)$ of $G$. These concepts were introduced by Gary Chartrand and first studied in [1]. In this note, we study a well-known class of trees, namely caterpillars.

A caterpillar $T$ is a tree of order 3 or more, the removal of whose leaves produces a path called the spine of $T$. A star is therefore a caterpillar with a trivial spine and a double star (a tree of diameter 3 ) is a caterpillar whose spine is the path $P_{2}$ of order 2. A caterpillar possessing an ID-coloring is therefore an ID-caterpillar. Here, we determine all those caterpillars that are ID-caterpillars and their possible ID-numbers. For this purpose, it is useful to present some results obtained in [1,2].

For an integer $t \geq 2$, the members of a set $S$ of $t$ vertices in a graph $G$ are called $t$-tuplets (twins if $t=2$ and triplets if $t=3$ ) if either (1) $S$ is an independent set in $G$ and every two vertices in $S$ have the same neighborhood or (2) $S$ is a clique, that is the subgraph $G[S]$ induced by $S$ is complete and every two vertices in $S$ have the same closed neighborhood.

Proposition 1.1. Let $G$ be a connected graph with twins $u$ and $v$. If $c$ is an ID-coloring of $G$, then $c(u) \neq c(v)$. Consequently, If $G$ is an ID-graph, then $G$ is triplet-free.

Proposition 1.2. Let $c$ be a red-white coloring of a connected graph $G$ where there is at least one vertex of each color. If $x$ is a red vertex and $y$ is a white vertex, then $\vec{d}(x) \neq \vec{d}(y)$.

Theorem 1.1. For a positive integer $r$, there exists a connected graph $G$ with $\operatorname{ID}(G)=r$ if and only if $r \neq 2$. 
Theorem 1.2. A nontrivial connected graph $G$ has $\operatorname{ID}(G)=1$ if and only if $G$ is a path.

Theorem 1.3. A connected graph $G$ of diameter 2 is an ID-graph if and only if $G=P_{3}$.

Since every caterpillar of maximum degree 5 or more contains triplets, the following observation is an immediate consequence of Proposition 1.1.

Observation 1.1. No caterpillar of maximum degree 5 or more is an ID-tree.

By Theorems 1.2 and 1.3 and Observation 1.1, we need only consider caterpillars of diameter 3 or more and maximum degree 3 or 4 . In this note, we present the following results on ID-caterpillars and their ID-numbers.

1. A caterpillar is an ID-caterpillar if and only if it is triplet-free.

2. If $T$ is a caterpillar of diameter 3 or more and maximum degree 3 , then $\operatorname{ID}(T)=3$.

3. If $T$ be a $k$-twin caterpillar (a caterpillar with exactly $k$ pairs of twins) of diameter 4 or more and maximum degree 4 , then $\max \{3, k\} \leq \operatorname{ID}(T) \leq k+3$. Furthermore, for each pair $(k, t)$ of integers where $k \geq 0$ and $t \in\{0,1,2,3\}$ such that $k+t \geq 3$, there is a $k$-twin caterpillar $T_{k, t}$ for which $\operatorname{ID}\left(T_{k, t}\right)=k+t$.

\section{Which caterpillars are ID-graphs?}

We begin with caterpillars of diameter 3 or more and maximum degree 3 .

Theorem 2.1. If $T$ is a caterpillar of diameter 3 or more and maximum degree 3 , then $T$ is an ID-caterpillar and $\operatorname{ID}(T)=3$.

Proof. Let $T$ be a caterpillar of diameter $d \geq 3$ and maximum degree 3. By Theorems 1.1 and 1.2, it suffices to show that $T$ has an ID-coloring with exactly three red vertices. If $d=3$, then $T$ is a double star of order 5 or 6 . Since these two double stars have ID-colorings with exactly three red vertices (as shown in Figure 1), we may assume that $d \geq 4$.
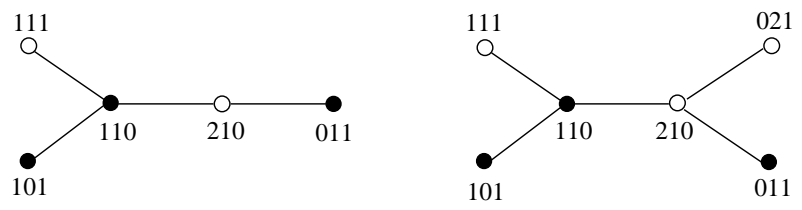

Figure 1: ID-colorings of two double stars.

Let $P=\left(v_{0}, v_{1}, \ldots, v_{d}\right)$ be a longest path in $T$, where $d=\operatorname{diam}(T) \geq 4$. For $1 \leq i \leq d-1$, if $\operatorname{deg} v_{i}=3$, then let $u_{i}$ be the end-vertex of $T$ that is not in $P$ and adjacent to $v_{i}$. Define a red-white coloring $c$ of $T$ by assigning the color red to $v_{i}$ if $i=0,1, d$ and the color white to the remaining vertices of $T$. This red-white coloring is illustrated for the caterpillar of diameter 8 in Figure 2. We show that $c$ is an ID-coloring of $T$. Let $x$ and $y$ be two distinct vertices of $T$. By Proposition 1.2, we may assume that $x$ and $y$ have the same color in $T$. Since (i) the first and the last coordinates of $\vec{d}\left(v_{0}\right)$ are both 1 , (ii) the first coordinate of $\vec{d}\left(v_{1}\right)$ is 1 and the last coordinate of $\vec{d}\left(v_{1}\right)$ is 0 , and (iii) the first coordinate of $\vec{d}\left(v_{d}\right)$ is 0 and the last coordinate of $\vec{d}\left(v_{d}\right)$ is 1 , it follows that the three red vertices $v_{0}, v_{1}$ and $v_{d}$ have distinct codes. Thus, we may assume that $x$ and $y$ are white vertices. Let $\vec{d}(x)=\left(a_{1}, a_{2}, \ldots, a_{d}\right)$ and $\vec{d}(y)=\left(b_{1}, b_{2}, \ldots, b_{d}\right)$.

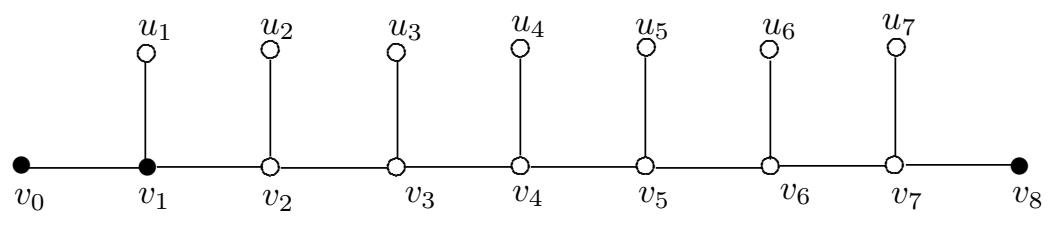

Figure 2: An ID-coloring of a caterpillar of diameter 8.

First, observe that for each vertex $z$ of $T$, the eccentricity of $z$ is $e(z)=\max \left\{d\left(z, v_{0}\right), d\left(z, v_{d}\right)\right\}$. Since $v_{0}$ and $v_{d}$ are red vertices of $T$, it follows that the $e(z)$ th coordinate of $\vec{d}(z)$ is the final coordinate of $\vec{d}(z)$ that is not 0 . Consequently, if $e(x) \neq e(y)$, say $e(x)<e(y)=s$, then $a_{s}=0$ but $b_{s} \neq 0$, which implies that $\vec{d}(x) \neq \vec{d}(y)$. Hence, we may assume that $e(x)=e(y)=s$. We consider three cases, according to the location of $x$ and $y$.

Case 1. $x=u_{i}$ and $y=u_{j}$ where $1 \leq i<j \leq d-1$. Since $e\left(u_{i}\right)=e\left(u_{j}\right)=s$ and $1 \leq i<j \leq d-1$, it follows that $e\left(u_{i}\right)=d\left(u_{i}, v_{d}\right)$ and $e\left(u_{j}\right)=d\left(u_{j}, v_{0}\right)$. Then $b_{s-1}=1\left(\right.$ since $\left.d\left(u_{j}, v_{1}\right)=s-1\right)$ and $a_{s-1}=0$. Hence, $\vec{d}(x) \neq \vec{d}(y)$. 
Case 2. $x=v_{i}$ and $y=v_{j}$ where $2 \leq i<j \leq d-1$. Since $e\left(v_{i}\right)=e\left(v_{j}\right)=s$ and $2 \leq i<j \leq d-1$, it follows that $e\left(v_{i}\right)=d\left(v_{i}, v_{d}\right)$ and $e\left(v_{j}\right)=d\left(v_{j}, v_{0}\right)$. Then $b_{s-1}=1$ (since $\left.d\left(v_{j}, v_{1}\right)=s-1\right)$ and $a_{s-1}=0$. Hence, $\vec{d}(x) \neq \vec{d}(y)$.

Case 3. $x=u_{i}$ where $1 \leq i \leq d-1$ and $y=v_{j}$ where $2 \leq i<j \leq d-1$. Since $e\left(u_{i}\right)=e\left(v_{j}\right)=s$, it follows that $y \in\left\{v_{i-1}, v_{i+1}\right\}$. If $y=v_{i-1}$, then $d\left(u_{i}, v_{d}\right)=d\left(v_{i-1}, v_{d}\right)=s=d-i+1$. Since $d\left(v_{i-1}, v_{1}\right)=i-2$ and $d\left(u_{i}, v_{1}\right)=i$, it follows that $a_{i-2}=0$ and $b_{i-2}=1$. Hence, $\vec{d}(x) \neq \vec{d}(y)$. If $y=v_{i+1}$, then $d\left(u_{i}, v_{0}\right)=d\left(v_{i+1}, v_{0}\right)=s=i+1$ and $d\left(u_{i}, v_{1}\right)=d\left(v_{i+1}, v_{1}\right)$. If $a_{s} \neq b_{s}$ or $a_{s-1} \neq b_{s-1}$, then $\vec{d}(x) \neq \vec{d}(y)$. Thus, we may assume that $a_{s}=b_{s}$ and $a_{s-1}=b_{s-1}$. Since $d\left(u_{i}, v_{d}\right)=d\left(v_{i+1}, v_{d}\right)+2$, it follows that $a_{s}=b_{s}=1$ and $a_{s-1}=b_{s-1}=1$. This implies that $b_{d-i-1}=1$, where $d-i-1=d\left(v_{i+1}, v_{d}\right)$, and $a_{d-i-1}=0$, implying that $\vec{d}(x) \neq \vec{d}(y)$.

We now consider triplet-free caterpillars of diameter at least 4 and maximum degree 4 . A triplet-free caterpillar with exactly $k$ pairs of twins is called a $k$-twin caterpillar. Figure 3 shows a 2 -twin caterpillar of diameter 4 and a 4 -twin caterpillar of diameter 5 . Next we show that every triplet-free caterpillar of diameter at least 4 and maximum degree 4 is an ID-caterpillar and present bounds for the ID-number of a $k$-twin caterpillar, which shows that its ID-number is one of four numbers (in terms of $k$ ).
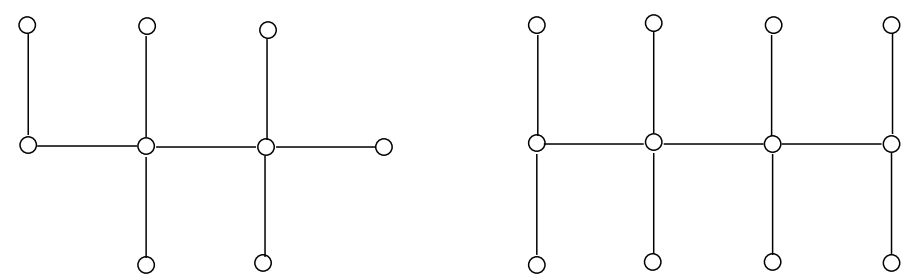

Figure 3: A 2-twin caterpillar and a 4-twin caterpillar.

Theorem 2.2. Let $T$ be a caterpillar of diameter $d \geq 4$ and maximum degree 4 . If $T$ is a $k$-twin for some positive integer $k$, then $T$ is an ID-caterpillar and

$$
\max \{3, k\} \leq \mathrm{ID}(T) \leq k+3 .
$$

Proof. Since $\operatorname{ID}(T) \geq \max \{3, k\}$ by Proposition 1.1 and Theorems 1.1-1.3, it remains to show that $\operatorname{ID}(T) \leq k+3$. Let $P=\left(v_{1}\right.$, $\left.v_{2}, \ldots, v_{d-1}\right)$ be the spine of $T$. For each integer $i$ with $1 \leq i \leq d-1$, let $L_{i}$ be the set of end-vertices of $T$ that are adjacent to $v_{i}$. Then $1 \leq\left|L_{i}\right| \leq 3$ for $i=1, d-1$ and $0 \leq\left|L_{i}\right| \leq 2$ for $2 \leq i \leq d-2$. We may assume that $\operatorname{deg} v_{2} \geq \operatorname{deg} v_{d-2}$. Define a red-white coloring $c$ of $T$ by

(1) assigning the color red to $v_{1}$, exactly one vertex $u_{1}$ in $L_{1}$, exactly one vertex $u_{d-1}$ in $L_{d-1}$, and exactly one vertex $u_{i}$ in $L_{i}$ if $\left|L_{i}\right|=2$ for $2 \leq i \leq d-2$ and

(2) assigning the color white to the remaining vertices of $T$.

Thus, for $1 \leq i \leq d-1$, the red end-vertex adjacent to $v_{i}$ (should it exist) is denoted by $u_{i}$ and the white end-vertex adjacent to $v_{i}$ (should it exist) is denoted by $w_{i}$. In particular, the red vertex $u_{1}$ is adjacent to the red vertex $v_{1}$ and the red vertex $u_{d-1}$ is adjacent to the white vertex $v_{d-1}$. Let $r$ denote the number of red vertices in $T$. Then

$$
r= \begin{cases}k+3 & \text { if }\left|L_{1}\right|=\left|L_{d-1}\right|=1 \\ k+2 & \text { if }\left\{\left|L_{1}\right|,\left|L_{d-1}\right|\right\}=\{1,2\} \\ k+1 & \text { if }\left|L_{1}\right|=\left|L_{d-1}\right|=2 .\end{cases}
$$

Thus, $r \geq 3$ if $k \in\{0,1,2\}$. It remains to show that $c$ is an ID-coloring of $T$. Let $x$ and $y$ be two distinct vertices of $T$. By Proposition 1.2, we may assume that $x$ and $y$ have the same color in $T$. Let $\vec{d}(x)=\left(a_{1}, a_{2}, \ldots, a_{d}\right)$ and $\vec{d}(y)=\left(b_{1}, b_{2}, \ldots, b_{d}\right)$. First, observe that for each vertex $z$ of $T$, the eccentricity of $z$ is $e(z)=\max \left\{d\left(z, u_{1}\right), d\left(z, u_{d-1}\right)\right\}$. Since $u_{1}$ and $u_{d-1}$ are red vertices of $T$, it follows that the $e(z)$ th coordinate of $\vec{d}(z)$ is the final coordinate of $\vec{d}(z)$ that is not 0 . Consequently, if $e(x) \neq e(y)$, say $e(x)<e(y)=s$, then $a_{s}=0$ but $b_{s} \neq 0$, which implies that $\vec{d}(x) \neq \vec{d}(y)$. Hence, we may assume that $e(x)=e(y)=s$. We consider two cases, according to whether $x$ and $y$ are both red or both white.

Case 1. $x$ and $y$ are both red. Observe that

- $u_{1}$ and $v_{1}$ are the only red vertices whose first coordinate is 1 and $e\left(u_{1}\right)>e\left(v_{1}\right)$;

- for $2 \leq i \leq d-1$, if $u_{i}$ exists, then the first coordinate of $\vec{d}\left(u_{i}\right)$ is 0 and $e\left(u_{d-1}\right)>e\left(u_{i}\right)$ for $2 \leq i \leq d-2$. 
Thus, we may assume that $x=u_{i}$ and $y=u_{j}$ where $2 \leq i<j \leq d-2$. Since $e\left(u_{i}\right)=e\left(u_{j}\right)=s$ and $2 \leq i<j \leq d-2$, it follows that $s=d\left(u_{i}, u_{d-1}\right)=d\left(u_{j}, u_{1}\right)$. Since (1) $d\left(u_{j}, v_{1}\right)=d\left(u_{i}, v_{d-1}\right)=s-1$, (2) $v_{1}$ is red and $v_{d-1}$ is white, and (3) $\operatorname{deg} v_{2} \geq \operatorname{deg} v_{d-2}$, it follows that $b_{s-1}>a_{s-1}$. Hence, $\vec{d}(x) \neq \vec{d}(y)$.

Case 2. $x$ and $y$ are both white. Observe that

- $w_{1}$ (should it exist) is the only white vertex whose whose code begins with 111

- $w_{d-1}$ (should it exist) is only white peripheral vertex whose code begins with 0 , and

- $w_{2}$ (should it exist) is the only white vertex whose whose code begins with 02 .

Thus, we may assume that $x, y \in\left\{w_{i}: 3 \leq i \leq d-2\right\} \cup\left\{v_{i}: 2 \leq i \leq d-1\right\}$. We consider three cases, according to the location of $x$ and $y$.

Subcase 2.1. $x=w_{i}$ and $y=w_{j}$ where $3 \leq i<j \leq d-2$. Since $e\left(w_{i}\right)=e\left(w_{j}\right)=s$ and $3 \leq i<j \leq d-2$, it follows that $s=d\left(w_{i}, u_{d-1}\right)=d\left(w_{j}, u_{1}\right)$. Since (1) $\operatorname{deg} v_{2} \geq \operatorname{deg} v_{d-2}$, (2) $v_{1}$ is red and $v_{d-1}$ is white, and (3) $d\left(w_{i}, v_{d-1}\right)=d\left(w_{j}, v_{1}\right)=$ $d\left(w_{j}, u_{2}\right)=s-1$ (should $u_{2}$ exist), we have $b_{s-1}>a_{s-1}$. Hence, $\vec{d}(x) \neq \vec{d}(y)$.

Subcase 2.2. $x=v_{i}$ and $y=v_{j}$ where $2 \leq i<j \leq d-1$. Since $e\left(v_{i}\right)=e\left(v_{j}\right)=s$ and $2 \leq i<j \leq d-1$, it follows that $s=d\left(v_{i}, u_{d-1}\right)=d\left(v_{j}, u_{1}\right)$. Since (1) $\operatorname{deg} v_{2} \geq \operatorname{deg} v_{d-2}$, (2) $v_{1}$ is red and $v_{d-1}$ is white, and (3) $d\left(v_{j}, v_{1}\right)=d\left(v_{j}, u_{2}\right)=s-1$ (should $u_{2}$ exist), we have $b_{s-1}>a_{s-1}$. Hence, $\vec{d}(x) \neq \vec{d}(y)$.

Subcase 2.3. $x=w_{i}$ where $3 \leq i \leq d-2$ and $y=v_{j}$ where $2 \leq i<j \leq d-1$. Since $e\left(w_{i}\right)=e\left(v_{j}\right)=s$, it follows that $y \in\left\{v_{i-1}, v_{i+1}\right\}$. If $y=v_{i-1}$ where $i \geq 3$, then $d\left(w_{i}, u_{d-1}\right)=d\left(v_{i-1}, u_{d-1}\right)=s$. Since (1) $\operatorname{deg} v_{2} \geq \operatorname{deg} v_{d-2}$ and (2) $d\left(v_{i-1}, v_{1}\right)=d\left(v_{i-1}, u_{2}\right)=i-2$ (should $u_{2}$ exist) and $d\left(w_{i}, v_{1}\right)=i$, it follows that $b_{i-2}>a_{i-2}=1$. Hence, $\vec{d}(x) \neq \vec{d}(y)$. If $y=v_{i+1}$, then $d\left(w_{i}, u_{1}\right)=d\left(v_{i+1}, u_{1}\right)=s$. Observe that if $z \in\left\{v_{p}, u_{p}: 1 \leq p \leq i\right\} \cup\left\{w_{p}: 1 \leq p \leq i-1\right\}$, then $d\left(w_{i}, z\right)=d\left(v_{i+1}, z\right)$. Let $t$ be the smallest integer with $i+1 \leq t \leq d-1$ such that $u_{t}$ exists (where it is possible that $t=d-1)$ and so $u_{t}$ is red. Since $d\left(w_{i}, u_{t}\right)=d\left(v_{i+1}, u_{t}\right)+2$ and $t-i=d\left(v_{i+1}, u_{t}\right)$, it follows that $b_{t-i}>a_{t-i}$. Hence, $\vec{d}(x) \neq \vec{d}(y)$.

By Theorem 2.2, if $T$ is a $k$-twin caterpillar, then $\max \{3, k\} \leq \operatorname{ID}(T) \leq k+3$. In fact, every integer between $\max \{3, k\}$ and $\leq k+3$ is realizable as the ID-number of some $k$-twin caterpillar, as we show next.

Theorem 2.3. For each pair $(k, t)$ of integers where $k \geq 0$ and $t \in\{0,1,2,3\}$ such that $k+t \geq 3$, there is a $k$-twin caterpillar $T$ for which $\operatorname{ID}(T)=k+t$.

Proof. We verify the following four statements.

1. For each integer $k \geq 0$, there exists a $k$-twin caterpillar $T$ with $\operatorname{ID}(T)=k+3$.

2. For each integer $k \geq 1$, there exists a $k$-twin caterpillar $T$ with $\operatorname{ID}(T)=k+2$.

3. For each integer $k \geq 2$, there exists a $k$-twin caterpillar $T$ with $\operatorname{ID}(T)=k+1$.

4. For each integer $k \geq 3$, there exists a $k$-twin caterpillar $T$ with $\operatorname{ID}(T)=k$.

We provide a complete proof for Statements 1 and 2 and provide an outline of a proof for Statements 3 and 4 .

First, we verify Statement 1. By Theorem 2.1, if $T$ is a caterpillar of diameter 3 or more and maximum degree 3, then $\operatorname{ID}(T)=3$. Thus, the statement is true for $k=0$ and so we may assume that $k \geq 1$. Let $T$ be a caterpillar of diameter $d=k+3 \geq 4$ and let $P$ be the spine of $T$ such that each end-vertex of $P$ is adjacent to exactly one end-vertex and each interior vertex of $P$ is adjacent to exactly two end-vertices. Thus, $T$ contains exactly $k=d-3$ twins. We show that $\operatorname{ID}(T)=d=k+3$. Let $\left(v_{0}, v_{1}, \ldots, v_{d}\right)$ be the longest path in $T$ and so $P=\left(v_{1}, v_{2}, \ldots, v_{d-1}\right)$ is the spine of $T$. For each integer $i$ with $2 \leq i \leq d-2$, let $u_{i}$ and $w_{i}$ be the two end-vertices of $T$ that are adjacent to $v_{i}$. Since $T$ contains exactly $d-3$ twins, it follows by Theorem 2.2 that $\operatorname{ID}(T) \leq d$. Thus, it remains to show that $\operatorname{ID}(T) \geq d$. Assume, to the contrary, that $\operatorname{ID}(T) \leq d-1$ and let $c$ be an ID-coloring of $T$ with exactly $\operatorname{ID}(T)$ red vertices. Since $u_{i}$ and $w_{i}$ are twins in $T$ for $2 \leq i \leq d-2$, we may assume that $c\left(u_{i}\right)$ is red and $c\left(w_{i}\right)$ is white. Since $d\left(v_{1}, z\right)=d\left(w_{2}, z\right)$ for each $z \in V(T)-\left\{v_{0}\right\}$, it follows that if $v_{0}$ and $v_{1}$ are both white, then $\vec{d}\left(v_{1}\right)=\vec{d}\left(w_{2}\right)$, which is impossible. Thus, at least one of $v_{0}$ and $v_{1}$ is red. If $v_{0}$ is white and $v_{1}$ is red, then $\vec{d}\left(v_{1}\right)=\vec{d}\left(u_{2}\right)$. Thus, $v_{0}$ must be red. Similarly, $v_{d}$ must be red. Hence, $\operatorname{ID}(T)=d-1$ and $v_{0}, v_{d}$, and $u_{i}$, where $2 \leq i \leq d-2$, are the $d-1$ red vertices of $T$. However then, $\vec{d}\left(v_{0}\right)=\vec{d}\left(v_{d-1}\right)$, for example, which is impossible. Therefore, $\operatorname{ID}(T)=d=k+3$ and so Statement 1 holds. 
To verify Statement 2, let $T$ be a caterpillar of diameter $d=k+4 \geq 5$ where $\left(v_{0}, v_{1}, \ldots, v_{d}\right)$ be the longest path in $T$ such that $\operatorname{deg} v_{i}=2$ for $i=1, d-2, d-1$ and $\operatorname{deg} v_{i}=4$ for $2 \leq i \leq d-3$. Thus, $T$ contains exactly $k=d-4 \geq 1$ twins. We show that $\operatorname{ID}(T)=d-2=k+2 \geq 3$. For each integer $i$ with $2 \leq i \leq d-3$, let $u_{i}$ and $w_{i}$ be the two end-vertices of $T$ that are adjacent to $v_{i}$. First, we show that $\operatorname{ID}(T) \geq k+2$. Let $c$ be an ID-coloring of $T$. Since $u_{i}$ and $w_{i}$ are twins in $T$ for $2 \leq i \leq d-3$, we may assume that $c\left(u_{i}\right)$ is red and $c\left(w_{i}\right)$ is white. Since $d\left(v_{1}, z\right)=d\left(w_{2}, z\right)$ for each $z \in V(T)-\left\{v_{0}\right\}$, it follows that if $v_{0}$ and $v_{1}$ are both white, then $\vec{d}\left(v_{1}\right)=\vec{d}\left(w_{2}\right)$, which is impossible. Thus, at least one of $v_{0}$ and $v_{1}$ must be red. Similarly, at least one vertex in $\left\{v_{d-2}, v_{d-1}, v_{d}\right\}$ must be red. Thus, $\operatorname{ID}(T) \geq k+2$.

Next, we show that $T$ has an ID-coloring with exactly $k+2$ red vertices. Define a red-white coloring $c$ of $T$ by (1) assigning the color red to $v_{0}, v_{d}$, and $u_{i}$ for $2 \leq i \leq d-3$ and (2) assigning the color white to the remaining vertices of $T$. Thus, $T$ has exactly $k+2$ red vertices. We show that $c$ is an ID-coloring of $T$. Let $x$ and $y$ be two distinct vertices of the same color in $T$, where $\vec{d}(x)=\left(a_{1}, a_{2}, \ldots, a_{d}\right)$ and $\vec{d}(y)=\left(b_{1}, b_{2}, \ldots, b_{d}\right)$. First, observe that for each vertex $z$ of $T$, the eccentricity of $z$ is $e(z)=\max \left\{d\left(z, u_{1}\right), d\left(z, u_{d-1}\right)\right\}$. Since $u_{1}$ and $u_{d-1}$ are red vertices of $T$, it follows that the $e(z)$ th coordinate of $\vec{d}(z)$ is the final coordinate of $\vec{d}(z)$ that is not 0 . Consequently, if $e(x) \neq e(y)$, say $e(x)<e(y)=s$, then $a_{s}=0$ but $b_{s} \neq 0$, which implies that $\vec{d}(x) \neq \vec{d}(y)$. Hence, we may assume that $e(x)=e(y)=s$. We consider two cases, according to whether $x$ and $y$ are both red or both white.

Case 1. $x$ and $y$ are both red. Since (1) $v_{0}$ and $v_{d}$ are the only red peripheral vertices and (2) $\vec{d}\left(v_{0}\right)$ begins with 001 , and $\vec{d}\left(v_{d}\right)$ begins with 000, we may assume that $x=u_{i}$ and $y=u_{j}$ where $2 \leq i<j \leq d-2$. Since $e\left(u_{i}\right)=e\left(u_{j}\right)=s$ and $2 \leq i<j \leq d-2$, it follows that $s=d\left(u_{i}, v_{d}\right)=d\left(u_{j}, v_{0}\right)$. Then $b_{s-1}=1$ and $a_{s-1}=0$. Hence, $\vec{d}(x) \neq \vec{d}(y)$.

Case 2. $x$ and $y$ are both white. Observe that

- $v_{d-2}$ is the only white vertex whose whose code begins with 02 ,

- $v_{d-1}$ is the only white vertex whose whose code begins with 10 ,

- for $2 \leq i \leq d-3$, the first coordinate of $\vec{d}\left(w_{i}\right)$ is 0 and for $1 \leq j \leq d-3$, the first coordinate of $\vec{d}\left(v_{j}\right)$ is 1 , which implies that $\vec{d}\left(w_{i}\right) \neq \vec{d}\left(v_{j}\right)$, and

- $e\left(v_{1}\right)>e\left(v_{i}\right)$ for $2 \leq i \leq d-3$, which implies that $\vec{d}\left(v_{1}\right) \neq \vec{d}\left(v_{i}\right)$.

Thus, we may assume that $x=w_{i}$ and $y=w_{j}$ where $2 \leq i<j \leq d-3$ or $x=v_{i}$ and $y=v_{j}$ where $2 \leq i<j \leq d-3$. First, suppose that $x=w_{i}$ and $y=w_{j}$ where $2 \leq i<j \leq d-3$. Since $e\left(w_{i}\right)=e\left(w_{j}\right)=s$ and $2 \leq i<j \leq d-3$, it follows that $s=d\left(w_{i}, v_{d}\right)=d\left(u_{j}, v_{0}\right)$. Then $b_{s-1}>a_{s-1}$ (since $\left.d\left(u_{j}, u_{2}\right)=s-1\right)$. Hence, $\vec{d}(x) \neq \vec{d}(y)$. Next, suppose that $x=v_{i}$ and $y=v_{j}$ where $2 \leq i<j \leq d-3$. Since $e\left(v_{i}\right)=e\left(v_{j}\right)=s$ and $2 \leq i<j \leq d-3$, it follows that $s=d\left(v_{i}, v_{d}\right)=d\left(v_{j}, v_{0}\right)$. Then $b_{s-1}>a_{s-1}$ (since $d\left(v_{j}, u_{2}\right)=s-1$ ). Hence, $\vec{d}(x) \neq \vec{d}(y)$.

Hence, $c$ is an ID-coloring of $T$ with exactly $k+2$ red vertices and so $\operatorname{ID}(T) \leq k+2$. Therefore, $\operatorname{ID}(T)=k+2$ and Statement 2 holds.

We now provide an outline of a proof of Statement 3. By Theorem 2.1, every caterpillar of diameter 3 or more and maximum degree 3 has ID-number 3 . Thus, if $T$ is a caterpillar of diameter 3 or more and maximum degree 3 such that each of two end-vertices of its spine is adjacent to two end-vertices, then $T$ has $k=2$ twins such that $\operatorname{ID}(T)=3$. Consequently, we may assume that $k \geq 3$. Let $T$ be a caterpillar of diameter $d=k+1 \geq 4$ and let $P$ be the spine of $T$ such that every vertex of $P$ is adjacent to exactly two end-vertices. Thus, $T$ contains exactly $k=d-1$ twins. We show that $\operatorname{ID}(T)=d=k+1$. Let $P=\left(v_{1}, v_{2}, \ldots, v_{d-1}\right)$ be the spine of $T$. For each integer $i$ with $1 \leq i \leq d-1$, let $u_{i}$ and $w_{i}$ be the two end-vertices of $T$ that are adjacent to $v_{i}$. Since $u_{i}$ and $w_{i}$ are twins in $T$ for $1 \leq i \leq d-1$, it follows by Proposition 1.1 that $\operatorname{ID}(T) \geq d-1$. Assume, to the contrary, that there is an ID-coloring of $T$ with exactly $d-1$ red vertices. We may assume that $u_{i}$ is red for $1 \leq i \leq d-1$. However then, $v_{1}$ and $v_{d-1}$ have the same code, for example, which is impossible. Therefore, $\operatorname{ID}(T) \geq d$. To show $I D(T) \leq d$, we define a red-white coloring $c$ of $T$ by (1) assigning the color red to $v_{1}$ and $u_{i}$ for $1 \leq i \leq d-1$ and (2) assigning the color white to the remaining vertices of $T$. Thus, $T$ has exactly $d$ red vertices. It can be shown that $c$ is an ID-coloring of $T$. Therefore, $\operatorname{ID}(T)=d=k+1$.

To provide an outline of a proof Statement 4 , let $T$ be a caterpillar of diameter $d=k+2 \geq 5$ and let $P=\left(v_{1}, v_{2}, \ldots, v_{d-1}\right)$ be the spine of $T$ such that each vertex of $P$ is adjacent to exactly two end-vertices except $v_{d-2}$ having degree 2 . Thus, $T$ contains exactly $k=d-2$ twins. We show that $\operatorname{ID}(T)=d-2=k$. For each integer $i$ with $1 \leq i \leq d-1$ and $i \neq d-2$, let $u_{i}$ and $w_{i}$ be the two end-vertices of $T$ that are adjacent to $v_{i}$. Since $T$ contains exactly $d-2$ twins, it follows that $\operatorname{ID}(T) \geq d-2$. To show that $\operatorname{ID}(T) \leq d-2$, we define a red-white coloring $c$ of $T$ by (1) assigning the color red to $u_{i}$ for $1 \leq i \leq d-3$ and $u_{d-1}$ and (2) assigning the color white to the remaining vertices of $T$. It can be shown that $c$ is an ID-coloring of $T$ with exactly $d-2$ red vertices. Therefore, $\operatorname{ID}(T)=d-2=k$. 


\section{Acknowledgments}

We are grateful to Professor Gary Chartrand for suggesting concepts and problems to us and kindly providing useful information on this topic. Furthermore, we thank the anonymous referees whose valuable suggestions resulted in an improved paper.

\section{References}

[1] G. Chartrand, Y. Kono, P. Zhang, Distance vertex identification in graphs, J. Interconnection Netw. 21 (2021) \#2150005.

[2] Y. Kono, P. Zhang, Vertex identification in trees, Discrete Math. Lett. 7 (2021) 66-73. 